\title{
Modelling of multifrequency IRMPD for laser isotope separation
}

\author{
AKSHAYA K NAYAK* and SISIR K SARKAR \\ Laser and Plasma Technology Division, BARC, Mumbai 400 085, India \\ e-mail: mipslptd@magnum.barc.ernet.in
}

\begin{abstract}
The process of infrared multiple photon dissociation (IRMPD) of molecules is of great fundamental importance and has practical significance, such as isotope separation etc. Unfortunately, a clear insight into the process has been hindered by the bewildering array of important variables affecting MPD. The dissociation probability $\gamma(\phi)$ i.e. the yield has been found to be a sensitive function of laser fluence $\phi$ along with numerous other parameters like laser frequency, gas pressure etc. We have shown that in single frequency IRMPD, an accurate quantitative characterization of the dissociation probability can be adequately expressed by a 'power law' model with two fitting parameters namely critical fluence, $\phi_{c}$ and multiphoton order, $m$. This model was exploited in analysing our MPD results on various systems. However, the small isotope shift encountered in heavy elements and the sticking phenomenon observed in small light molecules restrict respectively the separation factor and the dissociation yield. These problems can effectively be tackled by irradiation with multifrequency laser beams which can be chosen appropriately on the basis of spectroscopic features. Based on our success in single frequency model, multifrequency IRMPD is modelled by a functional form containing the product of power law terms for individual fluences on irradiation frequencies. This model is successfully benchmarked with our experimental results on multifrequency LIS of tritium. Such knowledge can be utilized for appropriate separation process design, evaluation and optimization.
\end{abstract}

Keywords. Multifrequency multiphoton dissociation; laser isotope separation; tritium separation.

\section{Introduction}

A most exciting discovery in nonlinear laser chemistry is the phenomenon of infrared multiple photon excitation (IRMPE) and dissociation (IRMPD) of molecules. ${ }^{1-4}$ In MPE or MPD a molecule could be highly excited and eventually dissociated through frequency selective absorption of tens of photons from an IR laser. The process is of great fundamental importance and has far reaching scientific and practical significance, such as isotope separation, purification of materials, synthesis of novel molecules and even mode-selective chemistry. Currently, the main thrust is aimed at developing a workable separation method for ${ }^{235} \mathrm{U}$, the fissile isotope of uranium needed to fuel light water reactors. In addition there is promising market for isotope of light elements such as D, T, ${ }^{6} \mathrm{Li},{ }^{10} \mathrm{~B},{ }^{13} \mathrm{C},{ }^{18} \mathrm{O},{ }^{33} \mathrm{~S}$ etc., some in connection with energy production and others as tracers in analytical, medical and environmental studies. Unfortunately, a clear insight into the process has been hindered by the bewildering array of important variables

\footnotetext{
*For correspondence
} 
affecting MPD. Therefore, the research in understanding the principles involved remains an ongoing endeavour.

Currently one of the viable enrichment method is provided by irradiation of a low pressure gas sample with a high intensity pulsed IR laser, causing MPD. For this often the laser beam has to be focused to obtain the necessary fluence for MPD and this causes a fluence variation over many orders of magnitude within the irradiation volume. The dissociation probability $\gamma(\phi)$, i.e. the yield has been found to be a sensitive function of laser fluence along with other parameters like laser frequency, gas pressure etc. To assess the spatially averaged data obtained in MPD experiments, an accurate quantitative characterization of the dissociation probability is necessary by a suitable deconvolution procedure.

Such accurate quantitative modelling of the fluence dependence of the fractional dissociation per pulse is necessary to attain high separation factors and high fractional yields at the same time. We have shown that for single frequency IRMPD ${ }^{5}$, the fractional dissociation per pulse, $q$ can be adequately expressed by a 'power law' model with two fitting parameters. This model was exploited in analysing our MPD results on various systems. ${ }^{6}$ It has been verified experimentally that the values of the parameter $n$ are nearly identical for both resonant and off-resonant excitation in several molecules. In such cases the fluence dependence of conversion, both for a desirable isotope and for an undesirable one, can be easily determined from a small number of data points. As a consequence, the model allows to determine readily the condition for desired fractional yields and separation factors.

However, the small isotope shift encountered in heavy elements and also the sticking phenomenon observed in small molecules ${ }^{7}$ restrict the separation factor and the dissociation yield respectively. These problems can effectively be tackled by irradiation with multifrequency laser beam which can be chosen appropriately on the basis of spectroscopic features. In absence of a model function, very large amounts of data in multidimensional space are necessary to find the fluence conditions for desired yields and separation factors. Even when sufficient data are gathered after considerable effort, it may be difficult to interpret the fluence dependence inductively and find the desired condition systematically. Here, we present a model for multifrequency IRMPD using a uniform transverse profiled and focused laser beam for an optically thin sample. The dissociation probability is expressed by a functional form based on the product of power law terms for individual fluences of irradiation frequencies. Then this model is applied to our experimental results on multifrequency laser isotope separation of tritium.

\section{Experimental}

We present briefly the experimental arrangement as it has been described in detail elsewhere. ${ }^{8,9}$ Trifluoromethane- $\mathrm{T}\left(\mathrm{CTF}_{3}\right)$ was prepared by hydrogen isotope exchange of $\mathrm{CHF}_{3}$ with tritiated water in the presence of dimethyl sulphoxide and $\mathrm{NaOH}$. Typically, the $\mathrm{CHF}_{3}$ samples used in our experiment had $0.2 \mathrm{ppm}$ of $\mathrm{CTF}_{3}$.

A commercial $\mathrm{CO}_{2}$ laser (Lumonics TEA103-2) was used for irradiation experiments. The $2.2 \mathrm{~m}$ long cavity of the laser (discharge cross section $=9 \mathrm{~cm}^{2}$ ) was formed with a Littrow mounted blazed grating and a Ge output mirror $(R=10 \mathrm{~m})$. Typically when the grating is tuned to resonate for a particular rotational line, the resonance condition may as well hold for a few neighbouring rotational lines provided the output mirror has a concave geometry. The low gain lines, owing to their smaller population inversion, 
saturate at the slower rate, thus resulting in an overall reduction in competition among themselves. Hence, even with a partial spatial overlap, a group of neighbouring low gain lines may simultaneously grow to give multifrequency output. Thus, by an appropriate tuning of the grating angle to satisfy the resonance condition, different sets of lines can be made to lase simultaneously. The pulse energy was measured by a pyroelectric joule meter and frequencies were measured by a spectrum analyser. The transverse profile of the laser beam was found to have uniform profile.

All irradiations were carried out at room temperature in a pyrex cell $(30 \mathrm{~cm}$ in length and $3.5 \mathrm{~cm}$ in diameter) equipped with polished $\mathrm{KCl}$ windows. Focussing of the laser beam was done at the centre of the cell by a $\mathrm{BaF}_{2}$ lens $(f=25 \mathrm{~cm})$.

Irradiated samples were cryogenically transferred to a radio-gas chromatograph (GC) for analysis. The radio-GC consisted of a commercial GC (Shimadzu GC-R1A) equipped with thermal conductivity detector (TCD) and an indigenously built proportional counter $(10 \mathrm{ml}, 3 \mathrm{kV})$ for the analysis of tritium bearing species. A Porapack Q column (80-100 mesh, $2 \mathrm{~mm}$ id, $2 \mathrm{~m}$ long) was used at $35^{\circ} \mathrm{C}$ with $\mathrm{He}$ carrier gas (flow rate $25 \mathrm{ml} / \mathrm{min}$ ) to separate the only photoproduct $\mathrm{C}_{2} \mathrm{~F}_{4}$ from the starting tritiated fluoroform. The depletion in $\mathrm{CTF}_{3}$ and $\mathrm{CHF}_{3}$ could be simultaneously measured by the counter and TCD respectively. The $\mathrm{C}_{2} \mathrm{~F}_{4}$ peak was also used to countercheck the $\mathrm{CHF}_{3}$ dissociation.

\section{Model for single frequency excitation}

In a typical MPD experiment, a laser beam propagates centrally and axially through a cylindrical cell and is focused in the centre of the cell, while keeping the fluence at the cell windows lower than the damage threshold (typically $3-5 \mathrm{~J} \mathrm{~cm}^{-2}$ for $\mathrm{KCl}$ or $\mathrm{KBr}$ windows). The schematic diagram of the focused beam geometry is shown in figure 1 . The experimental dissociation yield may be expressed in terms of reaction volume $V_{R}$, which is defined as the product of the observed cell averaged specific dissociation rate, $d$ and the cell volume $V_{\text {cell }}: V_{R}=d \times V_{\text {cell }}$. Now the data analysis consists of derivation of parameters specific to the molecule from the values of $V_{R}$ which are the result of both molecular effect and the details of the geometry of the focused laser beam used. Ideally, this would involve the determination of the molecular dissociation probability, $\gamma(\phi)$ as a function of the fluence $\phi$ which is related to $V_{R}$ by

$$
V_{R}=\int_{\text {cell }} \gamma(\phi) \mathrm{d} V
$$

At least four different models ${ }^{10-14}$ have been reported for such evaluation. We have shown that in single frequency IRMPD, such deconvolution can be effectively performed by a 'power law' model with two fitting parameters namely critical fluence, $\phi_{c}$ and order of the process, $n$.

$$
\begin{aligned}
q=\gamma(\phi) & =\left(\phi / \phi_{c}\right)^{n}, \text { for } \phi<\phi_{c} \\
& =1, \quad \text { for } \phi \geq \phi_{c} .
\end{aligned}
$$

where $q$ is the local dissociation probability function and $\phi_{c}$ is the critical fluence.

In this model, two important criteria such as optical thickness of the sample and transverse profile of the laser beam need to be considered. Since most LIS experiments 
involve excitation/dissociation of minor isotope, the sample can be treated as optically thin. Single transverse mode $\left(\mathrm{TEM}_{\mathrm{oo}}\right)$ laser provides Gaussian beam profile while multimode laser has uniform or flat-top beam profile. Therefore, this model in an optically thin medium can be evaluated depending on whether the laser beam is Gaussian or uniform. The transverse and axial distribution $\gamma$ and $\phi$ are shown in the figure 1 for both the beam profiles. Since our working laser beam profile is closer to uniform, here we present only the treatment for uniform beam profile.

The beam envelope in the reaction cell can be expressed by

$$
r^{2}=r_{f}^{2}\left(1+z^{2} / a^{2}\right)
$$

(a)

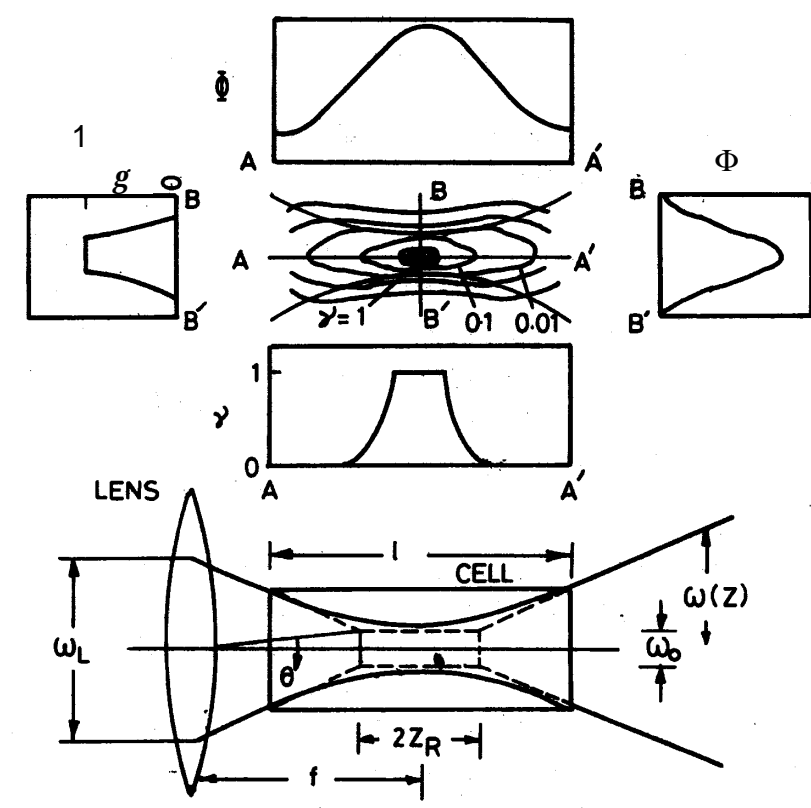

(b)

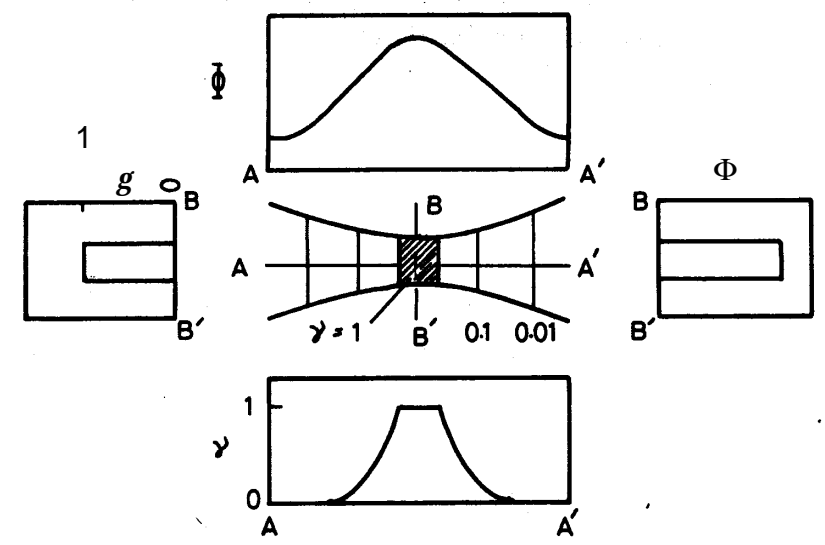

Figure 1. Schematic diagram of the focused beam geometry used in MPD experiments (middle). The transverse and axial distribution of $\gamma$ and $\phi$ are also shown for (a) Gaussian and (b) uniform profiled beams. 
where $a$ is the Rayleigh range, $r_{f}$ focal spot radius and $r$ is the radius of the irradiated zone at a distance $z$ from the focal point. The dimensionless reaction volume, $Y$ is defined as $\left(V_{R} / V_{f}\right)$ where the reaction volume $V_{R}$ normalized by the focal volume, $V_{f}=2 \pi_{f}^{2} a$. The dimensionless fluence $F$ is defined as the fluence $\phi_{0}$ at the beam-waist normalized by the critical fluence $\phi_{c}, F=\phi_{0} / \phi_{c}$. Denoting $\gamma(\phi)$ as $q$ and $2 L$ as the cell length

$$
V_{R}=\int_{\text {cell }} q \mathrm{~d} V=2 \int_{0}^{L} q \pi{ }^{2} \mathrm{~d} z .
$$

Under the condition of $\phi_{0}<\phi_{c}$, using expression for beam envelope for $r$ and the relation $\phi_{0} \pi_{f}^{2}=\phi \pi \sigma^{2}$, and putting $z / a=k$,

$$
Y=F^{n} \int_{0}^{L / a}\left(1+k^{2}\right)^{1-n} \mathrm{~d} k
$$

However, for $\phi_{0} \geq \phi_{c}$ the region of focusing beam envelope can be split into two regions; from $z=0$ to $z=l_{c}$ where $\phi \geq \phi_{c}$ and from $z=l_{c}$ to $z=L$ where $\phi<\phi_{c}$, and the reaction volume is given by:

$$
V_{R}=2\left(\int_{0}^{l_{c}} q \mathrm{~d} V+\int_{l_{c}}^{L} q \mathrm{~d} V\right) .
$$

Using expressions for $q$ in the two regions and replacing $z / a$ by $k$, for the case of $\phi_{0} \geq \phi_{c}$

$$
Y=(F-1)^{1 / 2}+1 / 3(F-1)^{3 / 2}+F^{n} \int_{(F-1)^{1 / 2}}^{L / a}\left(1+k^{2}\right)^{1-n} \mathrm{~d} k .
$$

First, we present some sample calculations to examine the sensitivity of fluence to the dissociation yield. This is to examine whether the pulse profile dependence predicted by the model actually holds to an acceptable degree for realistic cases. The result of sample calculations is presented in figure 2 which shows the $n$ and $\phi_{c}$ dependence. In a separate study ${ }^{6}$ the evaluation with a Gaussian beam profile was carried out and is incorporated in the above figure, for $n=3$. Comparing the two curves for $n=3$ it is evident that in the region of $F<1$ ( or $\phi<\phi_{c}$ ), the agreement is good, however the Gaussian beam treatment yields about a $10 \%$ higher value of critical fluence. From the above exercise, two important conclusions can be drawn. Firstly, it shows how the fluence dependence can change over to finally give a $3 / 2$ power dependence which is generally observed under tight focusing irradiation geometry. Secondly, the approximate independence of the pulse profile for fixed fluence condition indicate that it may not be very critical for data analysis if the laser sometimes unpredictably gets mode-locked.

After this fruitful exercise, the model was applied in analysing results of IRMPD of various systems investigated in our laboratory. Here, we present the results on tritium removal from contaminated reactor water. Table 1 gives the yields and selectivities for 
IRMPD of 8.5 Torr of $\mathrm{CHF}_{3} / \mathrm{CTF}_{3}$ with and without 20 Torr of Ar under single and quadruple frequency irradiation.

The important finding is almost a ten-fold increase in selectivity by distributing $1 \mathrm{~J}$ of energy of a single line over four lines. Distributing the total laser fluence over several frequencies led to a reduction of intensities on each frequency required for the individual excitation steps. In addition, one can in principle build up the selectivity by taking advantage of multiplication of the selectivity attained in the individual excitation step. ${ }^{15}$

\section{Model for multiple frequency excitation}

Based on our success on single frequency work, we present a model for such multifrequency IRMPD. In this model, the dissociation probability is expressed simply by the product of power law terms for individual fluences on irradiation frequencies, $\left(\Omega_{1}\right.$, $\left.\Omega_{2}, \ldots . \Omega_{N}\right)$. In this case the function $\gamma\left(\phi_{i}\right)$ can be given as

$$
\begin{array}{rlr}
q=\gamma\left(\phi_{i}\right) & =\left(\phi_{i} / \phi_{c i}\right)^{n}, & \text { for } \phi_{i}<\phi_{c i} \\
& =1, & \text { for } \phi_{i} \geq \phi_{c i},
\end{array}
$$

where $\phi_{i}$ is the fluence of the individual beam $\Omega_{i}(I=1,2, \ldots, N)$ and $\phi_{c i}$ is the critical fluence for each beam where the value of $\gamma$ saturates to unity. Then for a system with one dissociation channel, $q$ is expressed by the product of the functions $\gamma\left(\phi_{i}\right)$ :

$q=\prod_{i=1}^{N} \gamma\left(\phi_{i}\right)$, where $N$ is the total number of frequencies.

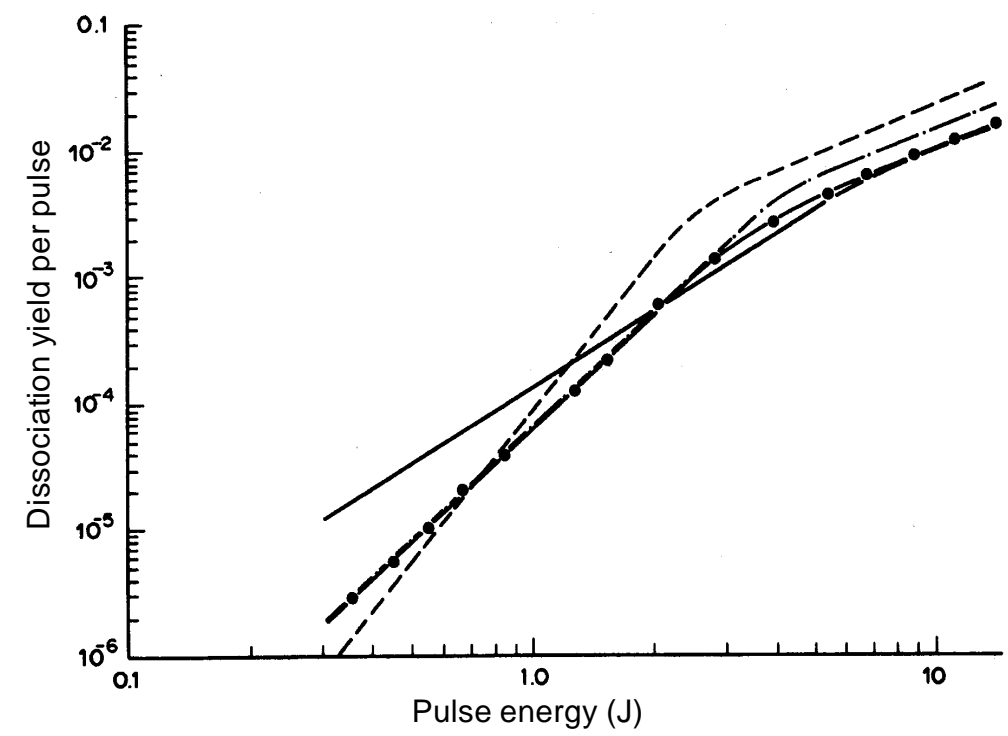

Figure 2. Model calculations of the dissociation yield with a uniform profiled laser pulse as a function of incident pulse energy; (---) $n=4, \phi_{c}=25 \mathrm{~J} \mathrm{~cm}^{-2},(-\cdots) n=3$, $\phi_{c}=40 \mathrm{~J} \mathrm{~cm}^{-2},(-) n=2, \phi_{c}=70 \mathrm{~J} \mathrm{~cm}^{-2}$. Also shown is the yield curve for a Gaussian laser beam with $n=3, \phi_{c}=44 \mathrm{~J} \mathrm{~cm}^{-2}$. 
Table 1. Yields and selectivities for IRMPD of 8.5 Torr of $\mathrm{CHF}_{3} / \mathrm{CTF}_{3}$ with and without 20 Torr of Ar under single and quadruple frequency irradiation.

\begin{tabular}{|c|c|c|c|c|c|c|c|}
\hline $\begin{array}{l}\text { Line/E } \\
(\mathrm{J})\end{array}$ & $\underset{(\mathrm{J})}{\mathrm{Line} / E}$ & $\underset{(\mathrm{J})}{\mathrm{Line} / E}$ & $\underset{(\mathrm{J})}{\mathrm{Line} / E}$ & $\begin{array}{c}P_{\mathrm{Ar}} \\
\text { (Torr) }\end{array}$ & $\begin{array}{c}d_{\mathrm{H}} \\
\left(\times 10^{5}\right)\end{array}$ & $\begin{array}{c}d_{\mathrm{T}} \\
\left(\times 10^{5}\right)\end{array}$ & $S(\mathrm{~T} / \mathrm{H})$ \\
\hline $\begin{array}{l}9 \mathrm{R}(8) \\
1.0\end{array}$ & - & - & - & - & 1.44 & $5 \cdot 38$ & 3.74 \\
\hline $\begin{array}{l}9 \mathrm{R}(8) \\
1.0\end{array}$ & - & - & - & 20 & $3 \cdot 60$ & $14 \cdot 57$ & $4 \cdot 05$ \\
\hline- & - & $\begin{array}{c}9 \mathrm{R}(12) \\
1.0\end{array}$ & - & - & $4 \cdot 40$ & $10 \cdot 60$ & $2 \cdot 41$ \\
\hline - & - & $\begin{array}{c}9 \mathrm{R}(12) \\
1.0\end{array}$ & - & 20 & $3 \cdot 43$ & $9 \cdot 09$ & $2 \cdot 65$ \\
\hline $\begin{array}{l}9 \mathrm{R}(8) \\
0.22\end{array}$ & $\begin{array}{c}\mathrm{R}(10) \\
0.26\end{array}$ & $\begin{array}{c}\mathrm{R}(12) \\
0.25\end{array}$ & $\begin{array}{c}\mathrm{R}(14) \\
0.27\end{array}$ & - & $0 \cdot 34$ & $4 \cdot 73$ & 13.91 \\
\hline $9 \mathrm{R}(8)$ & $\mathrm{R}(10)$ & $\mathrm{R}(12)$ & $\mathrm{R}(14)$ & 20 & $0 \cdot 28$ & $10 \cdot 73$ & $38 \cdot 32$ \\
\hline 0.22 & $0 \cdot 26$ & 0.25 & 0.27 & & & & \\
\hline 9R (20) & $\mathrm{R}(22)$ & $\mathrm{R}(24)$ & $\mathrm{R}(26)$ & - & $0 \cdot 38$ & 1.98 & $5 \cdot 21$ \\
\hline $\begin{array}{l}0 \cdot 25 \\
9 R(20)\end{array}$ & $\begin{array}{c}0 \cdot 30 \\
\mathrm{R}(22)\end{array}$ & $\begin{array}{c}0 \cdot 23 \\
\mathrm{R}(24)\end{array}$ & $\begin{array}{c}0 \cdot 22 \\
\mathrm{R}(26)\end{array}$ & & & & \\
\hline 0.25 & 0.30 & 0.23 & $0 \cdot 22$ & 20 & $0 \cdot 34$ & $4 \cdot 31$ & 12.69 \\
\hline
\end{tabular}

Generally speaking we have to consider the possibility of more than one channel for dissociation induced by different combination of frequencies. For instance, in two frequency $\left(\Omega_{1}, \Omega_{2}\right)$ irradiation the molecule may be dissociated through three channels: dissociation only by $\Omega_{1}$, only by $\Omega_{2}$ and by $\Omega_{1}$ plus $\Omega_{2}$. However, all these three channels may not be always significant. Expressing the dissociation yield for each of these three channels by $q_{1}, q_{2}$ and $q_{3}$, and because the dissociation through these channels occurs independently, $q$ can be related to $q_{i}$ 's by the equation:

$$
(1-q)=\left(1-q_{1}\right) \cdot\left(1-q_{2}\right) \cdot\left(1-q_{3}\right)
$$

For values of $q_{i}$ much smaller than unity, this becomes

$$
q=\sum_{j=1}^{M} q_{j},
$$

where $M$ is the number of dissociation channels and $q_{j}$ 's can be evaluated following the earlier prescription.

If the focal fluence of each individual frequencies is less than $\phi_{c i}$, then as in the single frequency case, since $q_{1}$ and $q_{2}$ are due to single frequency,

$$
\begin{aligned}
& Y_{1}=F_{1}^{n 1} \int_{0}^{L / a}\left(1+k^{2}\right)^{1-n 1} \mathrm{~d} k, \\
& Y_{2}=F_{2}^{n 2} \int_{0}^{L / a}\left(1+k^{2}\right)^{1-n 2} \mathrm{~d} k,
\end{aligned}
$$


where $F_{1}=\phi_{0,1} / \phi_{c, 1}$ and $F_{2}=\phi_{0,2} / \phi_{c, 2}$. The third channel is due to two frequencies $\Omega_{1}$ plus $\Omega_{2}$ and yield is given by

$$
Y_{3}=2 F_{11}^{n 1^{\prime}} \int_{0}^{L / a}\left(1+k^{2}\right)^{1-n 1^{\prime}-n 2^{\prime}} \mathrm{d} k,
$$

where $F_{11}=\phi_{0,1} / \phi_{c, 1}^{\prime}$ and $F_{22}=\phi_{0,2} / \phi_{c, 2}^{\prime}$. The primed quantities $\phi_{c, i}^{\prime}$ and $n_{i}^{\prime}$ designate the third dissociation channel. The addition of $Y_{1}, Y_{2}$ and $Y_{3}$ gives the normalized dissociation yield for two frequencies with three dissociation channels.

Now applying this model to our present investigation, four frequencies were used for excitation $\left(\Omega_{1}, \Omega_{2}, \Omega_{3}\right.$ and $\left.\Omega_{4}\right)$. The fluence in the irradiation zone was such that there would be negligible dissociation due to individual single frequency or with any combination of two frequencies. This leaves four channels of dissociation due to the combination of three frequencies and one channel due to all the four frequencies. Let the dissociation probabilities of these five channels be $q_{1}, q_{2}, q_{3}, q_{4}$ and $q_{5}$ respectively. Since the exciting frequencies were neighbouring laser lines, we can assume $q_{1}=q_{2}=q_{3}=q_{4}$. Hence overall $q$ can be approximated as $\left(4_{q_{1}}+q_{5}\right)$. Putting this in the expression for $V_{R}$ and solving for $Y$ we get,

$$
\begin{aligned}
& Y=12 F_{1}^{n 1} F_{2}^{n 2} F_{3}^{n 3} \int_{0}^{L / a}\left(1+k^{2}\right)^{1-n 1-n 2-n 3} \mathrm{~d} k+ \\
& 4 F_{11}^{n 1^{\prime}} F_{22}^{n 2^{\prime}} F_{33}^{n 3^{\prime}} F_{44}^{n 4^{\prime}} \int_{0}^{L / a}\left(1+k^{2}\right)^{1-n 1^{\prime}-n 2^{\prime}-n 3^{\prime}-n 4^{\prime}} \mathrm{d} k .
\end{aligned}
$$

A large number of parametric experiments need to be carried out to determine the values of $F_{i}, F_{i i}, n_{i}$ and $n_{i}{ }^{\prime}$ for evaluation of $Y$. Under the limited number of present experiments, further simplifications can be done by assuming $F_{i}$ and $F_{i i}$ 's to be equal since the total energy of $1 J$ is distributed almost equally and also by taking a single value $n\left(n^{\prime}\right)$ for all $n_{i}$ $\left(n_{i}{ }^{\prime}\right)$ for neighbouring four frequencies.

Table 2 represent the model parameters for IRMPD of 8.5 Torr of $\mathrm{CHF}_{3} / \mathrm{CTF}_{3}$ with 20 Torr of Ar under single and quadruple frequency irradiation. It can be seen that the

\begin{tabular}{|c|c|c|c|c|c|c|c|}
\hline Line & $\begin{array}{c}\phi_{\mathrm{cT}} \\
\left(\mathrm{J} / \mathrm{cm}^{2}\right)\end{array}$ & $\begin{array}{c}\phi_{\mathrm{cH}} \\
\left(\mathrm{J} / \mathrm{cm}^{2}\right)\end{array}$ & $n$ & $n^{\prime}$ & $\begin{array}{c}Y \\
\text { (Expt.) }\end{array}$ & $\begin{array}{c}Y \\
\text { (Theor.) }\end{array}$ & $\underset{(\mathrm{T} / \mathrm{H})}{S}$ \\
\hline 9R (8) & $\begin{array}{c}48 \\
-\end{array}$ & $\overline{144}$ & $\begin{array}{l}3 \cdot 0 \\
2 \cdot 5\end{array}$ & $\begin{array}{l}- \\
-\end{array}$ & $\begin{array}{l}5 \cdot 8 \\
1.43\end{array}$ & $\begin{array}{l}5.66 \\
1.45\end{array}$ & $4 \cdot 05$ \\
\hline 9R (12) & 66 & $\overline{150}$ & $\begin{array}{l}3 \cdot 0 \\
2 \cdot 5\end{array}$ & - & $\begin{array}{l}3.62 \\
1.37\end{array}$ & $\begin{array}{l}3.67 \\
1.36\end{array}$ & $2 \cdot 65$ \\
\hline 9R (8)-R (14) & 60 & - & $1 \cdot 5$ & $0 \cdot 6$ & $4 \cdot 2$ & $3 \cdot 6$ & \\
\hline & - & 175 & $1 \cdot 5$ & $0 \cdot 6$ & $0 \cdot 12$ & $0 \cdot 16$ & $36 \cdot 8$ \\
\hline $9 \mathrm{R}(20)-(26)$ & $\begin{array}{l}63 \\
-\end{array}$ & $\begin{array}{c}- \\
172\end{array}$ & $\begin{array}{l}1 \cdot 4 \\
1 \cdot 4\end{array}$ & $\begin{array}{l}0 \cdot 5 \\
0 \cdot 5\end{array}$ & $\begin{array}{l}1 \cdot 7 \\
0 \cdot 14\end{array}$ & $\begin{array}{l}1 \cdot 6 \\
0 \cdot 13\end{array}$ & $12 \cdot 3$ \\
\hline
\end{tabular}

Table 2. Model parameters for IRMPD of 8.5 Torr of $\mathrm{CHF}_{3} / \mathrm{CTF}_{3}$ with 20 Torr of Ar under single and quadruple frequency irradiation. 
yield and selectivity are satisfactorily evaluated using the model. The increase in critical fluence for the undesired species (in this case $\mathrm{CHF}_{3}$ ) in multiple frequency irradiation compared to single frequency case improved the selectivity of the process.

\section{Conclusions}

The present model has a simple form based on the model established for single frequency dissociation and at the same time has the flexibility to be applicable to multi-channel dissociation. Having successfully extracted the values of the fitting parameters for a specific molecule, the expected yield and selectivity can be accurately calculated for varying experimental conditions. Such knowledge can be utilized for appropriate design, evaluation and optimisation of laser separation process.

\section{References}

1. Ambartzumian R V and Letokhov V S 1977 In Chemical and biochemical applications of lasers (ed.) C B Moore (New York: Academic Press) vol. 3, p. 167

2. Schulz P A, Subdo A S, Krajnovitch D J, Kowk H S, Shen Y R and Lee Y T 1979 Annu. Rev. Phys. Chem. 30379

3. Letokhov V S 1983 In Nonlinear laser chemistry (Berlin: Springer Verlag)

4. Bagratasvili V N, Letokhov V S, Makarov A A and Raybov E A 1985 In Multiple photon infrared laser photophysics and photochemistry (London: Harwood)

5. Sarkar S K, Nayak A K, Parthasarathy V, Ramarao K V S and Mittal J P 1992 InfraredPhys. 33493

6. Sarkar S K, Nayak A K, Parthasarathy V, Ramarao K V S and Mittal J P 1992 Infrared Phys. 33505

7. Evseev A E, Letokhov V S and Puretzky A A 1985 Appl. Phys. B36 93

8. Nayak A K, Karve R S, Sarkar S K, Ramarao K V S and Mittal J P 1989 Appl. Phys. B49 139

9. Karve R S, Nayak A K, Sarkar S K, Ramarao K V S and Mittal J P 1989 Appl. Phys. B49571

10. Herman I P 1979 Opt. Lett. 4403

11. Marling J B, Herman I P and Thomas S J 1980 J. Chem. Phys. 725603

12. Nicol G R, Evans D K and McAlpine R D 1986 Appl. Phys. B39 29

13. Baldwin A C and Barker J R 1981 J. Chem. Phys. 743813

14. Herman I P, Takeuchi K and Makide Y 1989 In Laser applications in physical chemistry (ed.) D K Evans (New York: Dekker) p. 173

15. Letokhov V S and Mishin V I 1979 Opt. Commun. 29168 